discussion and risk of harm. In 4/22 cases there was no documentation of the clinical reasoning for a DNACPR decision. In 1 case the consultation led to potentially avoidable patient distress.

In 7 cases the patient lacked mental capacity to participate in a consultation; in 1 case it was felt it would cause too much harm to discuss the decision with their family.

Conclusion Patients and those important to them should be made aware of DNACPR decisions and these conversations recorded, including reasons for the decision. This audit however has highlighted ongoing variability in practice. Further training is needed in order to standardise practice and ensure high quality consultations around this sensitive topic are undertaken.

\section{PREPARING FUTURE DOCTORS FOR PROVIDING END OF LIFE CARE: CAN A SINGLE AFTERNOON TEACHING SESSION IMPROVE OUTCOMES?}

Alexandra Ramsey, Fiona Paterson, Ching Chung, Fiona McFatter. NHS Tayside, University of Dundee, Undergraduate Tayside Centre for General Practice

\subsection{6/bmjspcare-2018-ASPabstracts.44}

Background Caring for patients and their families at the end of life is a core requirement of the GMC Outcomes for Graduates, as well as NES and NHS Scotland Guidance. However, recent surveys have suggested that medical students feel unprepared for discussing and dealing with issues around death and dying.

Aims To pilot a communication and practical skills session around end of life care to final year medical students assessing whether having a single, focused teaching session enabled them to feel more prepared for their future roles as junior doctors as highlighted by national policy.

Methods Two teaching workshops were run in Spring 2017 at the University of Dundee, each lasting 2.5 hours in total. The sessions included communication with relatives about end of life care and care after death, as well as practical stations on end of life care prescribing and death verification/certification. Following each workshop, students were asked to complete a short survey assessing how useful they found the session and whether they felt more prepared in caring for the dying following it.

Results A total of 47 students attended both sessions and 41 $(87 \%)$ completed written feedback. All respondents agreed that the workshops helped them feel prepared to care for people who are dying and their relatives. Over 95\% of students felt that both communication skills stations were fairly or extremely useful, and over $90 \%$ of students felt that the practical stations were fairly or extremely useful.

Conclusions Our feedback shows that final year medical students view teaching around end of life care as an important part of their learning experience and that it would be welcomed if included in their training. Following this, we have integrated this teaching session into the curriculum with plans to further develop it by exploring alternative resources both locally and nationally.
18

DESCRIBING TREATMENT AIMS FOR PATIENTS APPROACHING THE END-OF-LIFE: MAPPING CONCEPTS FROM A SCOPING STUDY OF THE MEDICAL LITERATURE

SP Qureshi, A Dewar. University of Edinburgh

10.1136/bmjspcare-2018-ASPabstracts.45

Background Learning to care for patients approaching the end-of-life may be complicated by inconsistent language and lack of clear terms to discuss aims of treatments. To begin the process of developing a clear taxonomy for end-of-life care education, it is necessary to first establish the breadth of terms in use, and consider concepts they represent. This study aimed to map the scope of concepts utilised in discussion of treatment for patients approaching the end-of-life in medical literature.

Methods The authors undertook a scoping study of literature existing for the clinical education or professional development of medical doctors. This incorporated a systematic search of academic databases and non-database websites, and iterative development of inclusion criteria. Inductive qualitative content analysis was undertaken of the 269 included documents to examine terms, and their explicit and implicit meanings.

Results As analysis progressed, terms were grouped into one of three emerging over-arching categories according to the concepts represented. One of these major categories consisted of concepts for possible aims of care for patients approaching the end-of-life: Palliative care; End-of-life care; Advance care planning; Assessment of dying patients; Withdrawing treatment; Active treatment; Do Not Attempt Cardiopulmonary Resuscitation; Supportive Care; Preferred Place of Care; Medicalised Death; Conservative treatment; Advanced decision to refuse treatment; Curative change agents; Escalating medical care; Overtreatment. Interpretation and discussion of these concepts will be presented, including how they interrelate with or contradict one another.

Conclusions This study has delineated overlapping concepts in medical literature related to treatment aims for patients approaching the end-of-life. It has highlighted lack of consistent language. Further work is needed to develop clearly defined terms for teaching that can be widely understood and agreed upon.

\section{HIGH FIDELITY SIMULATION IN END OF LIFE CARE AS PART OF A SIMULATED WARD ROUND FOR FOUNDATION DOCTORS}

Lisa Boulstridge, Alicia Barnes, Victoria Knight. Burton Hospitals NHS Foundation Trust

10.1136/bmjspcare-2018-ASPabstracts.46

Background and Aims Foundation year doctors must achieve competency in palliative care outcomes. An end of life scenario in a high fidelity simulated ward round was designed and evaluated to gauge confidence levels in palliative care and quantify benefit derived.

Methods As part of trust induction Foundation Year 1 doctors (FY1) at Burton Hospitals NHS Trust undertake a simulated ward round with a palliative care scenario included. Newly 
qualified doctors assessed a patient with her distressed husband, commenced initial treatment and presented their case on the subsequent consultant post-take ward round. Training and orientation on local palliative care guidelines and resources followed. The benefit of this was assessed by anonymous questionnaire completed once the doctors had been in post for a month. They rated confidence in communication skills, managing symptoms, prescribing, decision making and holistic care approach.

Results $73 \%$ graded the exercise as highly beneficial. FY1 doctors confidence in the areas mentioned above ranged from 6.6 to 7.6 on a scale of $0-10$. The weakest area was discussing DNAR with patients, while the strongest area was confidence with end of life medications and doses. Other low scoring areas all focused around communicating either with the patient or their family, and making decisions about appropriate care (6.8-6.9). Areas of confidence focused around clinical aspects of care including managing symptoms, medications and assessing patient's needs (7.0-7.4).

Conclusion FY1s find communication skills and difficult conversations challenging. There is room for improvement across all areas analysed in order to achieve high confidence levels. We will continue to address these with two further high fidelity simulation sessions which will be assessed for benefit and improvement against the baseline confidence levels. These results will also be presented. Ultimately our aim is to help the new doctors achieve their competencies and provide high quality holistic care.

\section{SMALL GROUP RESEARCH IN THE EAST OF ENGLAND: A NOVEL APPROACH TO PALLIATIVE MEDICINE RESEARCH TRAINING}

Chloe Chin, Sarah Grove, Sarah Treaddell, Stephen Barclay, Anna Spathis. University of Cambridge, Cambridge University Hospitals NHS Foundation Trust, Arthur Rank Hospice, Garden House Hospice, Sue Ryder Thorpe Hall Hospice

\subsection{6/bmjspcare-2018-ASPabstracts.47}

Background Research is essential to advance the evidence base of Palliative Medicine. Few clinical trainees have the opportunity to become involved in research studies or to receive research training, which hampers trainees' ability to attain research competencies. Attending research courses may improve knowledge about research but are no substitute for participating in a study. Rotation of trainees also hinders completion of projects once started.

Aim To improve clinical trainee involvement in research to facilitate achievement of research competencies.

Development of novel approach A UK-wide survey of research training was undertaken. It found that over $75 \%$ of regions had no regular research training available. Only one region had a research training programme available for all Palliative Medicine trainees.

A programme was devised whereby East of England trainees were organised pragmatically by job location into a small research groups. Four out of twelve monthly study days were dedicated to research projects and training. Two clinical academic Palliative Medicine consultants provided supervision and met with all project groups twice yearly for advice and guidance.
Outcomes Since programme inception in 2015, outputs have included two poster and one oral presentations at national and international conferences, and one peer-reviewed journal publication. Three further projects are ongoing. Prior to the programme, research outputs were rare.

Participants report that project completion was facilitated by sharing workload amongst trainees, and by keeping projects location-based with newly rotating trainees providing continuity. The regional research meetings provided peer-to-peer support, the groups learning from each other's experiences.

Conclusion This novel approach has enhanced trainee research skills, experience and outputs. This form of research training programme could be readily adopted across the UK, being largely trainee-led: only intermittent support from consultants with an interest in research is needed.

\section{REDUCING HOSPICE ACQUIRED PRESSURE ULCERS: A THREE YEAR INITIATIVE}

Diana Sketchley. Weldmar Hospice Care, Joseph Weld Hospice

\subsection{6/bmjspcare-2018-ASPabstracts.48}

This poster outlines key implementations taken at Joseph Weld Hospice to reduce Hospice acquired pressure ulcers. Key areas for improvement were highlighted and improvements were made in assessment skills, documentation, education, Nurse/Doctor/Patient communication, reporting and hospice equipment.

Nursing assessment improvements were made through education including power point presentations: classification, risk factors and prevention. A pocket size pressure ulcer classification tool was designed for staff to have as an easy reference at the patient's bedside.

Documentation improvements included a redesign of the assessment windows on our electronic patient records to prompt accurate documentation.

Communication improvements in highlighting patients at high risk through a more detailed handover ensuring reports included any patients at risk of developing pressure ulcers.

Equipment was re-evaluated with patients need being assessed and utilised for our high risk patients and if in use air mattresses were sourced for hire to ensure pressure ulcer prevention. In May 2017 the hospice held a 'Comfort Appeal' to enable the hospice to fund new air mattresses. This exceeded our expectations raising funds within 2 months to buy 12 mattresses.

Results Year one, April 2014/2015 22 Patients developed pressure ulcers with 10 Pressure Ulcers reported as AVOIDABLE.

Year Two, April 2015/2016 23 Patients developed pressure ulcers but there was a huge reduction in AVOIDABLE pressure ulcers with only 2 reported.

Year Three, April 2016/2017 15 patients developed pressure ulcers but only 2 AVOIDABLE pressure ulcer reported.

Conclusion In making these key improvements there have been a significant reduction in hospice acquired pressure ulcers and remarkably the reduction in AVOIDABLE pressure Ulcers. The future hope for the hospice is to continue our best practice in minimising acquired pressure ulcers and abolish AVOIDABLE pressure ulcers. 